\title{
Tunnel magnetoresistance and spin transfer torque in magnetic tunnel junction with embedded nanoparticles
}

\author{
Niazbeck Useinov ${ }^{1, \star}$ \\ ${ }^{1}$ Institute of Physics, Kazan Federal University, Kazan 420008, Russia
}

\begin{abstract}
The theoretical model of spin-dependent transport in magnetic tunnel junctions (MTJ) containing magnetic or non-magnetic nanoparticle is developed. The dependences of tunnel magnetoresistance (TMR) and in-plane component of spin transfer torque (STT) on the applied voltage for various sizes of nanoparticles of the order of the mean free path of the conduction electron are calculated. The calculation is performed in the approximation of the ballistic transport of conduction electrons through the insulating layers of the MTJ and the nanoparticles.
\end{abstract}

\section{Introduction}

Currently the magnetic tunnel junction (MTJ) are widely studied in spintronics. The effects of tunnel magnetoresistance (TMR) and spin transfer torque (STT) in such nanostructures find their application in magnetic field sensors, spin current filters, non-volatile magnetoresistive randomaccess memory (MRAM, STT-MRAM), resonant tunnel diodes, spin transistors, etc.

The main goal of this work is to highlight the important conditions for dimension and voltage induced thresholds as well as demonstrate TMR and STT behaviors due to presence of nanoparticles (NPs) in insulator of MTJ. The MTJ-NPs (MTJ with embedded NPs) characteristics are similar to those in double barrier magnetic tunnel junctions (DMTJs), since the basic tunneling phenomena is the same. Double barrier MTJs are promising structures for MRAM applications due to better thermal stability, low noise and less critical current value for the switching in comparison to single barrier tunnel junctions (SMTJs) [1]. Moreover, both DMTJ and MTJ-NPs show a different spin-dependent quantum effects on ferromagnet/barrier and metallic (or magnetic) NP/barrier interfaces, which can be related to Coulomb blockade (CB), quantum well (QW) states, ballistic tunneling, resonant spin filtering and Kondo effect depending on middle layer thickness and applied voltage [2-7].

The basic tunneling approach has similar origin with the theory of giant magnetoresistance in point-like contacts [8] reproducing the limit of the point-like contact when NP touches top and bottom ferromagnetic (FM) layers. However, many aspects of the problem related to variation of barrier asymmetry, size distribution, quantization of the states and temperature dependence were not completely studied.

\footnotetext{
^e-mail: nuseinov@mail.ru
}

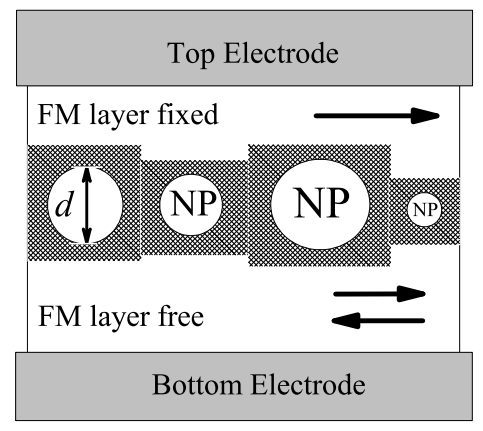

Figure 1. Schematic view of a magnetic tunnel junction with tunnel cells and nanoparticles (NP) of different sizes in an isolating middle layer (shaded). The arrows show the parallel (P) and antiparallel (AP) direction of the magnetizations of the free and fixed FM layers.

The present work is based on the assumption of the ballistic transport in MTJ-NPs, which were considered as a chain of the double barrier tunneling cells (TCs) connected in parallel, as shown in Fig. 1. Experimental works [6, 7] were considered as a data sources for the comparison with our results. In particular the MTJ-NPs was characterized by the average diameters of nanoparticles from $d=1.0 \pm$ $0.4 \mathrm{~nm}$ to $d=3.2 \pm 0.7 \mathrm{~nm}$.

\section{Ballistic transport of electrons through insulating layers and nanoparticles}

Magnetic tunnel junction with embedded nanoparticles was simulated as one hundred TCs connected in parallel $\left(N_{\text {tot }}=100\right)$. A few TCs are shown schematically in Fig. 1, each TC contains one NP. Ten fractions $f=1 . .10$ were considered with diameter $d^{(f)}$, and $\sum_{f} i_{f}=100$, 


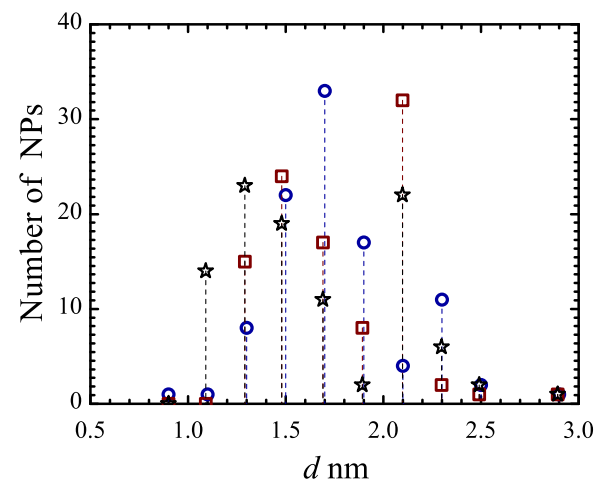

Figure 2. Distribution nanoparticles in MTJ with the set diameter $d$. The number nanoparticles with the given diameter in fraction is designated by corresponding signs with a line to an axis of abscisses. These numbers and signs correspond to calculations and the schedules presented further in drawings. The rings, rectangles and stars correspond to the simulation solid lines in Fig. 4, curve 1, 2 and 3.

where $i$ is a number of NPs. The fraction is defined as a set of TCs with the same diameter of NPs. The distribution of nanoparticles and their separation into fractions in MTJ, depending on their diameter is shown schematically in Fig. 2. Note that this distribution corresponds to the size of the NPs studied in [6].

The ballistic tunneling was considered as a basic transport approach. The spin-polarized current for each TC was defined as follows $[9,10]$ :

$$
I_{s, f}^{\mathrm{P}(\mathrm{AP})}=\frac{e^{2}}{h} \frac{\sigma_{f} k_{F, s}^{2} V}{2 \pi}\left\langle\cos \left(\theta_{s}\right) D_{s}^{\mathrm{P}(\mathrm{AP})}(V)\right\rangle,
$$

where $V$ is the applied voltage, $\theta_{s}$ is the incident angle which arises from conservation of the longitudinal projections of the $k$-vectors to the interfaces, $s=\uparrow, \downarrow$ is the spin index, $\sigma_{f}$ is an area of TC, $k_{F, s}$ is defined as a Fermi wavenumber of the top FM layer for positive voltage. The Eq. (1) consists integrals over the top incident electron trajectory angles $\theta_{s}$ and $\varphi$ and it is derived more accurate than in case WKB method, since the transmission coefficient $D_{s}$ was obtained as exact analytical solution for the double barrier system [11]. In our case $D_{s}$ is a function of $k_{F, s}, \theta_{s}$ and wavenumber through of the nanoparticle, $k_{n, s}^{(f)}$.

The wavenumber values for majority and minority electron bands were assumed as initial parameters of FM layers at zero voltage for all considered TCs as $k_{F, \uparrow}=$ $1.09 \AA^{-1}$ and $k_{F, \downarrow}=0.421 \AA^{-1}$ respectively. In present work the $k$-values are assumed as effective parameters which reflect the barrier transparency to filter out an electron wavefuctions and properties of spin bands at FM/barrier interfaces [12, 13].

To consider the positive voltage impact and spin filtering effect of NPs, electron wavenumbers in NPs and bottom FM layer were modified as follows:

$$
\begin{aligned}
& k_{n, s}^{(f)}(V)=\left[\left(k_{n}^{(f)}\right)^{2} \pm \frac{m_{\mathrm{eff}} \Delta}{\hbar^{2}}+c \frac{m_{\mathrm{eff}}}{m_{0}} \frac{V}{2}\right]^{1 / 2}, \\
& k_{F, s}(V)=\left(k_{F, s}^{2}+c V\right)^{1 / 2},
\end{aligned}
$$

where $c=2 m_{0} e / \hbar^{2} \cdot 10^{-18} \approx 26.25 \mathrm{~nm}^{-2} \mathrm{eV}^{-1}$ is the dimensional factor, $m_{\mathrm{eff}}$ and $m_{0}$ is the effective and free electron mass, $\Delta$ is the exchange energy which determines the difference between electron spin states, and the plus or minus sign corresponds to $s=\uparrow$ or $s=\downarrow$. In the case of a nonmagnetic NP, electronic states are degenerate in spin.

The transverse wavenumber $k_{n, s}^{(f)}$ in NP is the key parameter, since the result of integration (1) is very sensitive to its value. The related choice of initial $k_{n, s}^{(f)}$ was varied from $0.1 \AA^{-1}$ to $0.6 \AA^{-1}$. The lowest $k_{n, s}^{(f)}$ values corresponds to NPs distribution with greatest averaged NP diameter: $d_{\mathrm{av}}=\frac{1}{N_{\mathrm{tot}}} \sum i_{f} d^{(f)}$. The quantum well solution, which was used as initial approach of $k_{n}^{(f)}$ distribution over $d$, is presented below:

$$
k_{n}^{(f)}=\pi n / d^{(f)}-\Phi^{(f)},
$$

where correction term $\Phi^{(f)}$ that places in $k_{n}^{(f)}$ aside from ideal QW solution corresponding to the real systems; index $n$ is obtained as an integer number corresponding to QW discrete levels. The Eq. (3) is simplified quantization rule, $2 k_{n} d+\Phi_{B}+\Phi_{T}=2 \pi n$, and term $\Phi_{B(T)}$ is the phase change of the electron wave function on interfaces $[3,4]$. However, tunneling behavior at $d>1.8 \mathrm{~nm}$ can be different due to increased NP volume, and then NP reveals more bulk-assisted properties, whereas degree of quantization is vanished.

In our study tunnel magnetoresistance in the MTJ-NPs is determined by the ratio: $\mathrm{TMR}=\left(I^{\mathrm{P}}-I^{\mathrm{AP}}\right) / I^{\mathrm{AP}} \times$ $100 \%$, where

$$
I^{\mathrm{P}(\mathrm{AP})}=\sum i_{f} \cdot\left(I_{\uparrow, f}^{\mathrm{P}(\mathrm{AP})}+I_{\downarrow, f}^{\mathrm{P}(\mathrm{AP})}\right),
$$

and each term $I_{s, f}^{\mathrm{P}(\mathrm{AP})}$ was calculated by (1). The tunneling current are passing through insulator between NPs, was considered as negligible impact. The barriers thicknesses in symmetric MTJ-NPs were fixed as $L=1.0 \mathrm{~nm}$, the height of potential barriers $U_{B}=1.2 \mathrm{eV}$, the effective electron mass $m_{\mathrm{eff}}=0.8 m_{0}$ for NPs, $m_{\mathrm{eff}}=0.4 m_{0}$ for barriers and $m_{\mathrm{eff}}=m_{0}$ for FM layers are assumed.

One of important dynamic MTJ properties of the magnetic distortion is spin transfer torque (STT) effect $[14,15]$. The STT is determined by vector which consists of parallel $T_{\|}$and perpendicular $T_{\perp}$ components in relation to interfaces. The parallel component $T_{\|}$vanishes at zero voltage, while at finite voltage it lies in the plane of FM layer interfaces triggering additional magnetization fluctuation. The value of $T_{\perp}$ is determined by interlayer exchange interaction, which isn't vanished at zero voltage, $T_{\perp} \neq 0$. Only $T_{\|}$component will be considered below, since we assumed in our case $T_{\|}>T_{\perp}$. Parallel STT component is calculated as difference between spin current densities $\mathfrak{J}^{\mathrm{P}(\mathrm{AP})}$, Ref.[14, 15]:

$$
T_{\|}=\frac{\sin (\gamma)}{2}\left(\mathfrak{J}^{\mathrm{P}}-\mathfrak{J}^{\mathrm{AP}}\right),
$$

where $\mathfrak{J}^{\mathrm{P}(\mathrm{AP})}=\hbar\left(J_{\uparrow}^{\mathrm{P}(\mathrm{AP})}-J_{\downarrow}^{\mathrm{P}(\mathrm{AP})}\right) / 2 e, \quad \gamma$ is an angle between FM layers magnetizations; $J_{s}^{\mathrm{P}(\mathrm{AP})}=I_{s}^{\mathrm{P}(\mathrm{AP})} / S$ is the charge current density. Here $S=\frac{\pi}{4} \sum_{f=1 . .10} i_{f}\left[d^{(f)}\right]^{2}$ is the total active cross-section area of the MTJ-NPs. 


\section{Resuits and discussion}

\subsection{Tunnel magnetoresistance}

Now we will consider several effects that characterize MTJ-NPs, when the magnetizations of the FM layers are localized in the contact plane. Abnormal behavior of TMR shown in Fig. 3, which reproduces peak-like TMR dependencies and their suppression at zero voltages $V \simeq 0$. We shall denote such domains of TMR with the index zero $\mathrm{TMR}_{0}$. The calculations show that MTJ-NPs increase the electron transmission coefficient and create resonance conditions providing a quantization of the conductance. This is shown by curve 1 in Fig. 3 which was obtained for MTJ with non-magnetic NPs at values of $k_{n}=0.4508 \AA^{-1}$ and $d_{\mathrm{av}}=2.6 \mathrm{~nm}$. The Fig. 3 shows that $\mathrm{TMR}_{0}$ can take even negative values. Similar TMR dependencies were previously demonstrated in the experimental works [6,7]. In our calculations this corresponds to states with small wavenumber: $k_{n} \approx 0.1 \div 0.36 \AA^{-1}$. For example, $\mathrm{TMR}_{0}$

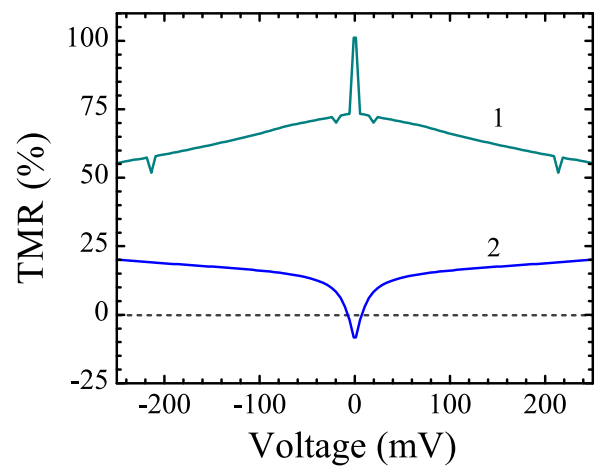

Figure 3. Bias voltage dependence of TMR for the magnetic tunnel junctions with embedded non-magnetic nanoparticles: curve 1 shows peak-like TMR for the case of wavenumber $k_{n}=0.4508 \AA^{-1}$ of the nanoparticle, curve 2 shows negative $\mathrm{TMR}_{0}=-8 \%$ for $k_{n}=0.115 \AA^{-1}$.

becomes negative for $k_{n}=0.115 \AA^{-1}$ and $d_{\mathrm{av}}=2.6 \mathrm{~nm}$, see Fig. 3, curve 2. Such values of $\mathrm{TMR}_{0}$ can also be observed experimentally due to the influence of interfaces and the uneven thickness of the barriers, as well as due to the influence of the anisotropic shape of the nanoparticles, when the wavenumber is described by the formula (3). The calculation of the transmission coefficient $D_{s}^{\mathrm{P}(\mathrm{AP})}$ for small $k_{n, s}$ also includes solutions in which the tunnel transparency at the expense of open trajectories in the allowed angles $\theta_{s}$. The influence of temperature on the value of TMR in our model is not discussed, since in the derivation of the expression for the current $I_{s, f}^{\mathrm{P}(\mathrm{AP})}$ is assumed that the operating temperatures are much less than the characteristic Fermi temperature $E_{F} / k_{B}$, where $k_{B}$ is the Boltzmann constant.

The tunneling magnetoresistance as a function of the applied voltage is strongly modified at $n=1,2$ in Fig. 4 . Also the Fig. 4 show the experimental data fitting which was made according to the presented theoretical approach (1) and (2) for magnetic NPs. The $\mathrm{TMR}_{0}$ curve corresponds to $n=1$ and $\Phi=0.1 \mathrm{~nm}^{-1}$. The diameter distribution, Fig. 2 (rings), is close to experimental data [6],

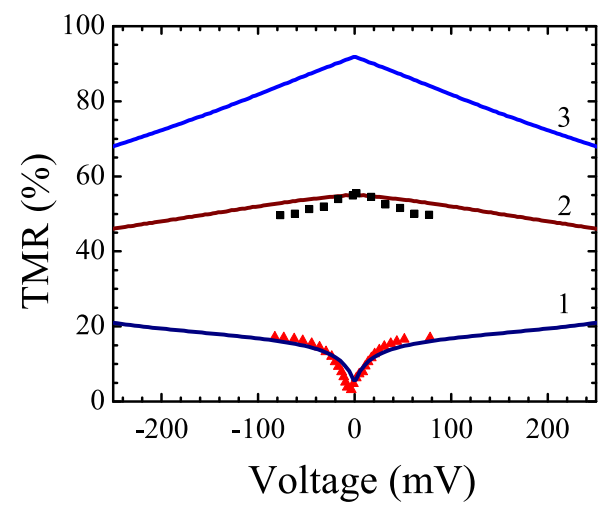

Figure 4. TMR- $V$ dependencies with related diameter distributions: the curve 1 with reduced $\mathrm{TMR}_{0}$ is shown for $n=1$, $\Phi=0.1 \mathrm{~nm}^{-1}$ in comparison to exp. data [6] with diameter of NPs distribution similar to the Fig. 2 (rings); curve 2 and 3 at $n=2, \Phi=0$ for distributions rectangles and stars of the Fig. 2, respectively.

where dip-like TMR behavior was observed only at low temperatures, $T \approx 2.5 \mathrm{~K}$. The gradual changes TMR variations show the initially suppressed TMR at low voltages (curve 1), and then the dome-like behavior (curve 3), depending on the distribution of diameters shown in Fig. 2 (see rings and stars). All initial $k_{n}^{(f)}$ are different for each fraction according to (3). The formation of the high $\mathrm{TMR}_{0}$, with dome-like behavior, follows to the fraction with lowest $k_{n}^{(f)}$ (curve 2) that is similar to the case $n=1$. The lowest $k_{n}^{(f)}$ values corresponds to NPs distribution with greatest averaged NP diameter $d_{\mathrm{av}}$. The calculated TMR curves satisfactorily reproduces experimental data at this case (see Fig. 4 and Fig. S6 in Ref.[6]), however, TMR behavior in relation to parameter $d$ is opposite to experimental observations for $d_{\mathrm{av}}>1.8 \mathrm{~nm}$. Thus, this can be more rational explanation when $k_{n, s}^{(f)}$ also increases with $d_{\mathrm{av}}$. It is assumed, that there is a size threshold at $d_{\mathrm{av}} \approx 1.8 \mathrm{~nm}$ between quasi-1D and bulk-assisted states in the middle conducting layer, and thus, quantization rule (3) have to be changed.

\subsection{Spin transfer torque}

The parallel component $T_{\|}$was calculated for the case when the magnetization directions of the upper and lower FM layers are perpendicular to each other, i.e. $\gamma=\pi / 2$. Note that $T_{\|}$has the maximum value when the direction of the magnetic moment of the nanoparticle follows the direction (or coincides with the direction) of the magnetization of the lower FM layer. Due to the identical determination of the spin-polarized current in single-barrier MTJ and in MTJ with nanoparticles during ballistic tunneling of electrons, in both cases the parallel STT component was calculated in a similar way using the equation (5).

Figure 5 shows the dependence of $T_{\|}$on the applied voltage for MTJ with nanoparticles in different states (see curves 1-3) and for a single-barrier MTJ (see curve 4). The values of the parameters at which the dependences were obtained correspond to Fig. 3. One can see a fast 


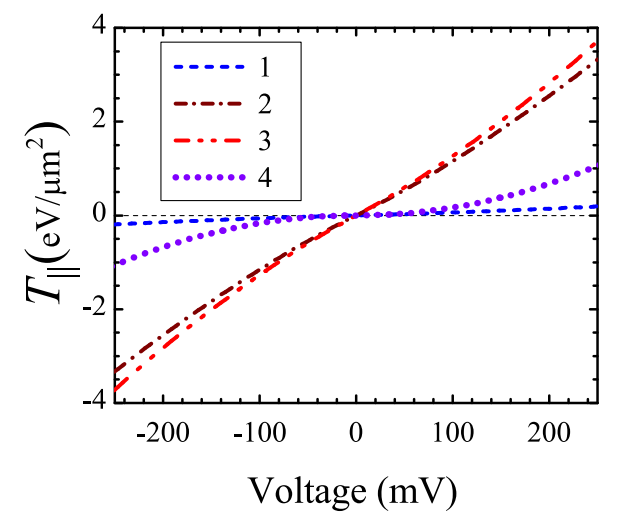

Figure 5. Dependences of the parallel component of the STT on the applied voltage for MTJ with nanoparticles (curves 1 and 2) and single-barrier MTJ (curve 4). The values of the parameters for which the curves were obtained correspond to Fig. 3. Curve 3 shows the dependence of the $T_{\|}$on $V$ for magnetic nanoparticles, when $k_{n, \uparrow(\downarrow)}=0.5026 \pm 0.0025 \AA^{-1}$.

and almost linear increase of $T_{\|}$for MTJ with nanoparticles having averaged diameter $d_{\mathrm{av}}=1.25 \mathrm{~nm}$ (see curve 2). For MTJ with magnetic nanoparticles, the values of $T_{\|}$ show the greatest increase with the voltage (see curve 3 ). In addition, the calculations show that if the thickness of the MTJ with non-magnetic NPs is $t=3,25 \mathrm{~nm}$, where $t=2 L+d$, then $T_{\|}$is comparable to its value in a singlebarrier MTJ with a barrier thickness of $1.0 \mathrm{~nm}$ (see curve 4). This value of $T_{\|}$can be even greater in the case of a magnetic NP, see curve 3 in Fig. 5.

Thus, we come to the conclusion that in the case of MTJ with magnetic nanoparticles, the large value of the parallel component STT can increase the fluctuations of two quantities: the magnetic moment of the nanoparticle and the magnetization of the soft magnetic FM layer (without taking into account the influence of temperature). As a result, this effect can also reduce the switching value of the critical current in the memory cell operating on the spin moment transfer effect, which agrees with the experimental data; see, for example, the work [16].

\section{Conclusion}

The development of the theory of spin-polarized electron transport in the process of quantization in magnetic NPs is based on the use of quantum mechanical and quasiclassical methods. Using the notion of two-barrier magnetic tunnel junctions with quantum wells made it possible to calculate the conductivity and reproduce qualitatively the TMR anomalies demonstrated in the experiments [6, 7]. Low electronic states in quantum wells and small values of wave vectors in NPs play an important role in observing the quantized conductivity regime.
The TMR anomalies such as peak behavior or suppression of their maximum value (negative TMR values) at low voltages are obtained and explained under the assumption of the ballistic tunneling mode based on the values of the wave vectors $k_{n, s}$ of the NPs and in view of their distribution along the diameters.

The above theoretical model also predicts a rather large effect of the spin transfer torque in magnetic tunnel junctions with built-in NPs. This indicates that in such systems it is possible to reduce the critical current density for switching the magnetization of the FM layers. The calculations showed that the contacts studied are complex systems in which, for certain parameters, various dependences (in particular, resonance) of the TMR on the applied voltage can be realized, as well as an energetically effective STT leading to a switching of the magnetization of the FM layer.

\section{References}

[1] G.Q. Yu, Z. Diao, J.F. Feng, H. Kurt, X.F. Han, J. M. D. Coey, Appl. Phys. Let. 98, 112504 (2011)

[2] T.V. Pham, S. Miwa, D. Bang, T. Nozaki, et al., Solid State Commun. 183, 18 (2014)

[3] Y. Wang, Z.Y. Lu, X.G. Zhang, X.F. Han, Phys. Rev. Let. 97, 087210 (2006)

[4] T. Nozaki, N. Tezuka, K. Inomata, Phys. Rev. Let. 96, 027208 (2006)

[5] P. Sheng, F. Bonell, S. Miwa, T. Nakamura, et al., Appl. Phys. Rev. 102, 032406 (2013)

[6] H. Yang, S. Yang, S.S.P. Parkin, Nano Lett. 8, 340 (2008)

[7] D. Ciudad, Z.C. Wen, A.T. Hindmarch, E. Negusse, et al., Phys. Rev. B. 85, 214408 (2012)

[8] L.R. Tagirov, B.P. Vodopyanov, K.B. Efetov, Phys. Rev. B. 63, 104428 (2001)

[9] N.Kh. Useinov, L.R. Tagirov, Physics Procedia 75, 995 (2015)

[10] A.N. Useinov, L.X. Ye, N.K. Useinov, T.H. Wu, C.H. Lai, Sci. Rep. 5, 18026 (2015)

[11] A.N. Useinov, J. Kosel, N.K. Useinov, L.R. Tagirov, Phys. Rev. B. 84, 085424 (2011)

[12] X.G. Zhang, W.H. Butler, Phys. Rev. B. 70, 172407 (2004)

[13] W.H. Butler, Sci. Technol. Adv. Mater. 9, 014106 (2008)

[14] I. Theodonis, N. Kioussis, A. Kalitsov, M. Chshiev, W. Butler, Phys. Rev. Lett.97, 237205 (2006)

[15] A. Kalitsov, W. Silvestre, M. Chshiev, J Velev, Phys. Rev. B. 88, 104430 (2013)

[16] Z. Diao, A. Panchula, Y. Ding, et al., Appl. Phys. Lett. 90, 132508 (2007). 